\title{
Propriétés Antifongiques Des Légumineuses Médicinales De Côte d'Ivoire : Cas De Crotalaria retusa L. (Fabaceae) Sur La Croissance in vitro De Phytophthora sp. Et Fusarium solani, Deux Champignons Phytopathogènes
}

\begin{abstract}
Doga Dabé
Laboratoire de Biotechnologie Végétale et Microbiologie Environnementale,

UMRI Sciences agronomiques et Génie Rural, Institut National

Polytechnique Félix HOUPHOUËT-BOIGNY (INP-HB), Yamoussoukro,

Côte d'Ivoire

\section{Zirihi Guédé Noël}

Laboratoire de Botanique, UFR Biosciences,

Université Félix Houphouët-Boigny, Abidjan, Côte d'Ivoire
\end{abstract}

\section{Zézé Adolphe}

Laboratoire de Biotechnologie Végétale et Microbiologie Environnementale,

UMRI Sciences agronomiques et Génie Rural, Institut National

Polytechnique Félix HOUPHOUËT-BOIGNY (INP-HB), Yamoussoukro,

Côte d'Ivoire

doi: 10.19044/esj.2016.v13n3p371 URL:http://dx.doi.org/10.19044/esj.2016.v13n3p371

\begin{abstract}
The high cost of fungicides, the international markets standards for agricultural product quality, the environmental issues and consumer health discourage to the application of chemical control. The strategy is therefore to develop alternative solutions, less expensive and addresses environmental concerns. This study was conducted to evaluate the fungicidal potency of Crotalaria retusa L. on Phytophthora sp. and Fusarium solani, two phytopathogenic fungi. This plant was selected after an ethnobotanical study conducted in the sub-prefecture of Lauzoua in Côte d'Ivoire. It is considered to be the most widely used in the treatment of microbial diseases. The phytochemical screening of this legume species was carried out, and showed the presence of saponosides, sterols, tannins, alkaloids, flavonoids, polyphenols, quinones and polyterpenes. The sensitivity of these two phytopathogenic fungi strains was tested in vitro in presence of the aqueous extract of Crotalaria retusa L. by using double dilution method on PDA medium (Potato Dextrose Agar). The minimum inhibitory concentration
\end{abstract}


(MIC) of spawn growth was $1.56 \mathrm{mg} / \mathrm{ml}$ for both fungi. The minimum fungicide concentration (CMF) was $6.25 \mathrm{mg} / \mathrm{ml}$ for Phytophthora $s p$ and $12.50 \mathrm{mg} / \mathrm{ml}$ for Fusarium solani. The presence of chemical compounds in this plant justifies its antifungal activity and its traditional use areas.

Keywords: Antifungal properties, Crotalaria retusa L., Fusarium solani, Phytophthora sp., Lauzoua, Côte d'Ivoire

\section{Résumé}

Le coût élevé des fongicides, les exigences des marchés internationaux en termes de qualité des produits agricoles, les préoccupations environnementales et la santé des consommateurs sont autant d'éléments qui ne favorisent pas l'application de la lutte chimique. La stratégie est donc de développer une méthode peu onéreuse et compatible avec les préoccupations environnementales. Cette étude a été conduite afin d'évaluer le pouvoir fongicide de Crotalaria retusa L. sur Phytophthora sp et Fusarium solani, deux champignons phytopathogènes. Cette plante a été sélectionnée à l'issue d'une étude ethnobotanique conduite dans la Sous-préfecture de Lauzoua en Côte d'Ivoire. Elle passe pour être la plus utilisée dans le traitement des maladies microbiennes. Le tri phytochimique réalisé sur cette plante a mis en évidence la présence des saponosides, des stérols, des tanins, des alcaloïdes, des flavonoïdes, des polyphénols, des quinones et des polyterpènes. La sensibilité de ces deux souches de champignons phytopathogènes a été testée in vitro en présence de l'extrait aqueux de Crotalaria retusa L. selon la méthode de double dilution sur milieu PDA (Potato Dextrose Agar). La concentration minimale inhibitrice (CMI) de la croissance mycélienne a été obtenue à $1,56 \mathrm{mg} / \mathrm{ml}$ pour les deux champignons. La concentration minimale fongicide (CMF) a été obtenue à $6,25 \mathrm{mg} / \mathrm{ml}$ pour Phytophthora $s p$. et à $12,50 \mathrm{mg} / \mathrm{ml}$ pour Fusarium solani. La présence des composés chimiques dans cette plante justifie son activité antifongique et son utilisation en milieu traditionnel.

Mots clés : Crotalaria retusa L., Fusarium solani, Phytophthora sp., propriétés antifongiques, Lauzoua, Côte d'Ivoire

\section{INTRODUCTION}

L'agriculture constitue une des bases de l'économie en Afrique subsaharienne notamment en Côte d'Ivoire. Malheureusement, elle est soumise à de nombreuses contraintes parmi lesquelles l'on peut citer la pression parasitaire. De nombreuses cultures vivrières et de rente font l'objet d'attaque des parasites dont les plus connus sont les champignons phytopathogènes. Parmi ceux-ci, figurent les espèces du genre Fusarium et 
Phytophthora. Les espèces du genre Fusarium s’attaquent aux cultures telles que la tomate (Hibar et al., 2007), le gombo (Drame, 2004), le riz (Zehhar et al.,2006), le blé (Lauzon et al., 2007), le bananier (Moore et al.,1995) et le cotonnier (Assigbetsé et al., 1990). Des études effectuées en 2015 dans trois zones de production de banane plantain en Côte d'Ivoire (Azaguié, Tiassalé et Anyaman) ont mis en évidence la présence des foyers de fusariose et dont l'agent causal a été Fusarium solani. La fusariose est donc une maladie responsable du flétrissement ou de la pourriture des racines et des collets des plantes. Dans certains cas, surtout chez les plantes céréalières, les graines peuvent être impropres à la transformation et à la consommation.

Par ailleurs, la pourriture brune des cabosses est une maladie très préjudiciable du cacaoyer. L'agent causal est Phytophthora sp. Au niveau mondial, cette maladie engendre des pertes de production de l'ordre de $30 \%$ (Lass, 1985). La Côte d'Ivoire, premier producteur mondial de cacao, avec plus de 40 \% de l'offre n’échappe pas à cette affection. Plusieurs espèces de Phytophthora peuvent être à l'origine de la maladie. Ce pathogène peut engendrer dans certains pays, la perte de la totalité de la production de cacao (Flood, 2006). En Côte d'Ivoire, la pourriture brune des cabosses prend de plus en plus de l'ampleur (Kouamé, 2006). En effet, le coût élevé des fongicides, les exigences des marchés internationaux en termes de qualité des produits agricoles, les préoccupations environnementales et la santé des consommateurs sont autant d'éléments qui ne favorisent pas l'application de la lutte chimique. La stratégie adoptée est le développement d'une méthode peu onéreuse et compatible avec les préoccupations environnementales. Il s'avère donc nécessaire d'explorer d'autres voies pour la protection des cultures contre les parasites fongiques. La lutte basée sur l'utilisation d'extraits végétaux pourrait constituer une réponse adéquate, économiquement et culturellement viable pour les agriculteurs africains.

Une étude ethnobotanique conduite à Lauzoua en Côte d'Ivoire a permis d'identifier 11 légumineuses parmi lesquelles Crotalaria retusa L. passe pour être la plus sollicitée dans les traitements des maladies microbiennes. Le but de notre travail est d'évaluer le pouvoir fongicide de Crotalaria retusa L. sur deux champignons modèles (Phytophthora sp. et Fusarium solani), deux champignons phytopathogènes économiquement importants.

\section{MATERIEL ET METHODES}

\section{1. MATERIEL}

\section{1. 1. Matériel végétal}

Le matériel végétal est constitué de feuilles de Crotalaria retusa L. (Figure 1). 


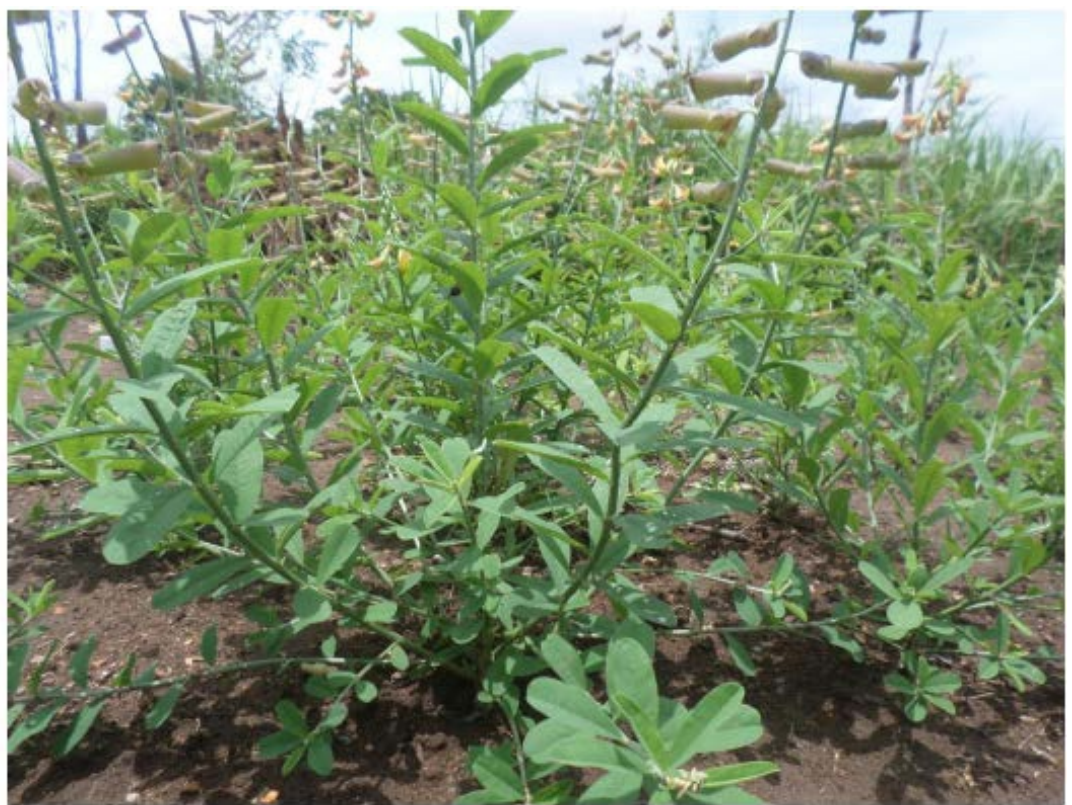

Figure 1 : Photo de Crotalaria retusa L. (photo Doga 2015)

\section{1. 2. Matériel fongique}

Le matériel fongique est représenté par deux souches de champignons phytopathogènes (Phytophthora sp. et Fusarium solani). Phytophthora sp. provient du Laboratoire de Phytopathologie et Biologie Végétale et Fusarium solani provient du Laboratoire de Biotechnologie Végétale et Microbiologie Environnementale. Ces deux Laboratoires appartiennent à l'Institut National Polytechnique Félix HOUPHOUËT-BOIGNY de Yamoussoukro (Côte d'Ivoire).

\section{2. Méthodes}

\section{2. 1. Sélection de la plante}

Une enquête ethnobotanique a été réalisée dans la Sous-préfecture de Lauzoua dans le Département de Guitry (Région de Lôh-Djiboua, Sud de la Côte d'Ivoire). Neuf (9) localités ont été visitées (8 villages : Adahidougou, Dougodou, Zogognigbo, Petit Bouaké, Lauzoua île, Lauzoua carrefour, Kikékou et Gobléko) et un campement (Gboto). Au cours de cette enquête, 35 tradipraticiens ont été interrogés. La méthode d'étude a été l'entretien semi-structuré. Cet entretien a eu lieu directement en langue Dida avec les autochtones et en Français ou par interprète pour les allogènes. A l'issue de l'enquête, 11 légumineuses ont été recensées et, une cinquantaine de recettes médicamenteuses a été cataloguée. Il ressort de cette enquête que Crotalaria retusa $\mathrm{L}$. entre dans la préparation de plusieurs types de médicaments prescrits dans le traitement des maladies microbiennes. Elle est très sollicitée 
dans le traitement des maladies de la peau. Cette plante a donc été choisie pour évaluer son activité antifongique sur des champignons phytopathogènes.

\section{2. 2. Mise en poudre du matériel végétal}

Les feuilles de Crotalaria retusa L. ont été récoltées au cours de l'enquête ethnobotanique. Ensuite, elles ont été lavées, découpées, puis séchées à l'ombre à la température ambiante pendant deux semaines. Après, elles ont été rendues en poudre fine grâce à un broyeur électrique de type IKA Labortechnik (type MFC). La poudre obtenue constitue le broyat qui servira à l'extraction du principe actif.

\section{2. 3. Extraction aqueuse et séchage du principe actif}

Cinquante (50) grammes de la poudre des feuilles de Crotalaria retusa L. ont été macérés dans un litre d'eau distillée à l'aide d'un mixer (Blender) pendant trois fois 3 min selon la méthode de Zirihi et al. (2003). L'homogénat obtenu a été filtré successivement deux fois sur du coton hydrophile et une fois sur du papier filtre Wathman $3 \mathrm{~mm}$. Le filtrat recueilli a été évaporé à l'étuve à $40^{\circ} \mathrm{C}$. La poudre ainsi obtenue est l'extrait aqueux de couleur marron foncé.

\section{2. 4. Tri phytochimique de l'extrait aqueux de Crotalaria retusa $\mathrm{L}$.}

Le tri phytochimique a été effectué afin de mettre en évidence quelques grands groupes de métabolites secondaires retrouvés dans les plantes. Les stérols, les polyterpènes, les polyphénols, les tanins, les flavonoïdes, les quinones, les saponosides et les alcaloïdes ont été recherchés. Le criblage a été fait suivant des réactions colorées selon la méthode de Bruneton (2009) et Mangambu et al. (2014). Les résultats sont marqués par des symboles suivants : ++ (abondant) et + (présence).

\section{2. 5. Mesure du taux d'inhibition de la croissance mycélienne}

Pour tester l'effet de l'extrait végétal sur la croissance des champignons, sept concentrations ont été retenues $(12,50 \mathrm{mg} / \mathrm{ml}, 6,25$ $\mathrm{mg} / \mathrm{ml}, 3,12 \mathrm{mg} / \mathrm{ml}, 1,56 \mathrm{mg} / \mathrm{ml}, 0,78 \mathrm{mg} / \mathrm{ml}, 0,39 \mathrm{mg} / \mathrm{ml}$ et $0,19 \mathrm{mg} / \mathrm{ml}$ ). Ces concentrations ont été définies selon la méthode de la double dilution de liaison géométrique de raison $1 / 2$ (Zirihi et al., 2003 ; Ahon et al., 2011). Les différentes quantités de l'extrait correspondant aux différentes concentrations déterminées, ont été ajoutées au milieu PDA (Potato Dextrose Agar) avant solidification $\left(40-50{ }^{\circ} \mathrm{C}\right)$. Ce milieu a été préalablement stérilisé à l'autoclave à $120^{\circ} \mathrm{C}$ pendant $20 \mathrm{mn}$. Le témoin n'a subi aucun amendement avec l'extrait. Après homogénéisation, ce mélange a été coulé à $40{ }^{\circ} \mathrm{C}$ dans des boîtes de pétri de $90 \mathrm{~mm}$ de diamètre. 
Un explant de $6 \mathrm{~mm}$ de diamètre de chaque champignon âgé de 7 jours, a été prélevé au niveau du front de croissance du champignon dans la boîte de culture et a été placé au centre géométrique de la boîte de Pétri sur le milieu solidifié. Les boîtes de Pétri ensemencées, ont été scellées avec du film adhésif et mises en incubation à l'étuve à $25 \pm 2{ }^{\circ} \mathrm{C}$ pendant 7 jours. Pour tous les essais, trois répétitions par traitement ont été faites.

Le taux d'inhibition de la croissance radiale mycélienne a été mesuré quotidiennement comparativement au témoin. Ce taux a été calculé selon la formule de Leroux et Credet (1978).

$\mathrm{T}(\%)=((\mathrm{D}-\mathrm{d}) / \mathrm{D}) \times 100$

$\mathrm{T}$ : taux d'inhibition

D : croissance mycélienne dans les boîtes témoins

D : croissance mycélienne dans les boîtes essais

Les boîtes dans lesquelles aucune croissance mycélienne n’a été visible à l'œil nu, ont été ouvertes. Puis, les explants de champignon ont été prélevés et ensemencés à nouveau dans de nouvelles boîtes de Pétri contenant le milieu PDA sans extrait végétal. Ces nouvelles boîtes ont été mises en incubation pendant 7 jours à la température ambiante $\left(27^{\circ} \mathrm{C}\right)$. Au terme de l'incubation, si une croissance mycélienne est observée, l'extrait est déclaré fongistatique. Dans le cas contraire, il est dit fongicide.

La détermination du taux d'inhibition de la croissance mycélienne de chaque souche fongique a donc permis de définir la concentration minimale inhibitrice (CMI) et la concentration minimale fongicide (CMF). La CMI est la plus petite concentration à partir de laquelle il n’y a aucune croissance. La CMF est la plus petite concentration à partir de laquelle aucune croissance mycélienne n'est observée dans les nouvelles boîtes de Pétri où les explants ont été réensemencées.

\section{RESULTATS}

\section{1. Sélection de la plante}

L'investigation ethnobotanique a permis d'identifier 11 légumineuses dans la pharmacopée du peuple de Lauzoua (Tableau I) dont Crotalaria retusa L. a été la plus citée (25,71\%). Diverses maladies sont traitées par ces plantes (Tableau II). Les principales maladies sont les infections et le paludisme. Crotalaria retusa L. a été celle qui traite plus d'affection, surtout les maladies microbiennes. Les modes de préparation des médicaments sont la décoction, la pulvérisation, la torréfaction et le pétrissage. Les modes d'administration sont la boisson, l'application locale, la purge, le bain et les instillations (oculaires et nasales). 
Tableau I : Quelques légumineuses utilisées dans la pharmacopée de Lauzoua

\begin{tabular}{llll}
\hline Espèces de légumineuses & \multicolumn{1}{c}{ Familles } & $\begin{array}{c}\text { Nombre de } \\
\text { praticiens }\end{array}$ & $\begin{array}{c}\text { Fréquence de } \\
\text { citation (\%) }\end{array}$ \\
\hline Cassia tora & Caesalpiniaceae & 2 & 5,71 \\
Desmodium gangeticum & Fabaceae & 4 & 11,43 \\
Crotalaria retusa & Fabaceae & $\mathbf{9}$ & $\mathbf{2 5 , 7 1}$ \\
Mucana pruriens & Fabaceae & 3 & 8,57 \\
Schrankia leptocarpa & Mimosaceae & 2 & 5,71 \\
Indigofera hirsuta & Fabaceae & 7 & 20 \\
Vigna sinensis & Fabaceae & 3 & 8,57 \\
Indigofera spicata & Fabaceae & 1 & 2,86 \\
Desmodium velutinum & Fabaceae & 8 & 22,85 \\
Cassia occidentalis & Caesalpiniaceae & 4 & 11,43 \\
Indigofera suffruticosa & Fabaceae & 6 & 17,14 \\
\hline
\end{tabular}

Tableau II : Pathologies soignées par ces légumineuses

\begin{tabular}{cc}
\hline Légumineuses & Pathologies soignées \\
\hline $\begin{array}{c}\text { Indigofera hirsuta L. } \\
\text { Indigofera suffruticosa } \\
\text { M. }\end{array}$ & $\begin{array}{c}\text { Paludisme, ictère, ulcère d'estomac, infections urinaires, } \\
\text { intestinales, fièvre infantile, parasitoses } \\
\text { inflammations, fièvre infantile, épilepsie }\end{array}$ \\
\hline $\begin{array}{c}\text { Crotalaria retusa L. } \\
\text { fièvre, conjonctivite, infection vaginale, parasitoses, intestinales, } \\
\text { vomissements }\end{array}$ \\
\begin{tabular}{cc}
$\begin{array}{c}\text { Desmodium velutinum } \\
\text { (Willd.) DC. }\end{array}$ & $\begin{array}{c}\text { Paludisme, ictère, maux de ventre, brûlures, fièvre infantile, } \\
\text { céphalées, douleur dentaire, vomissements, menace } \\
\text { d'avortement }\end{array}$ \\
\hline $\begin{array}{c}\text { Cassia tora L. } \\
\text { Cassia occidentalis L. }\end{array}$ & Maux de ventre, dermatose, céphalées, gonorrhée, maux d'yeux, \\
otite, paludismes, paludisme, dermatose, gonorrhée, ictère, infection \\
ovarienne, toux, ulcères, fièvre
\end{tabular} \\
\hline $\begin{array}{c}\text { Mucana pruriens (L.) } \\
\text { DC. }\end{array}$ & Paludisme, varicelle, infection ovarienne, vers intestinaux \\
\hline $\begin{array}{c}\text { Schrankia leptocarpa } \\
\text { DC. }\end{array}$ & Paludisme, maux de ventre, enflures \\
\hline $\begin{array}{c}\text { Desmodium } \\
\text { gangeticum (L.) DC. }\end{array}$ & Maux de ventre, paludisme, fièvre, céphalées, diarrhée, douleur \\
\hline $\begin{array}{c}\text { Indigofera spicata } \\
\text { Forssk }\end{array}$ & Fièvre \\
\hline Vigna sinensis L. & Ulcère d'estomac, toux \\
\hline
\end{tabular}

\section{2. Tri phytochimique}

La méthode de tri phytochimique utilisée, a indiqué la présence des principaux grands groupes chimiques dans la plante. Ces groupes chimiques sont consignés dans le tableau III. Les saponosides, les stérols, les tanins, les alcaloïdes et les polyterpènes ont été les métabolites secondaires les plus abondants dans cette légumineuse. 
Tableau III : Tri phytochimique de l'extrait aqueux de Crotalaria retusa L.

\begin{tabular}{|c|c|c|c|c|c|c|}
\hline \multirow{2}{*}{$\begin{array}{l}\text { Groupes } \\
\text { chimiques }\end{array}$} & \multirow[t]{2}{*}{ Sapo. } & \multirow[t]{2}{*}{ Flavo. } & \multirow[t]{2}{*}{ Polyph. } & \multirow{2}{*}{$\begin{array}{l}\text { Stérols, } \\
\text { polyterp. }\end{array}$} & Tanins & \multirow[t]{2}{*}{ Alcaloïdes } \\
\hline & & & & & & \\
\hline
\end{tabular}

Légumineuse

C. retusa $++\quad+$

$\begin{array}{lllll}++ & ++ & ++ & +\end{array}$ $++$ + : Présence ; ++ : Abondant,

Sapo : Saponosides, Flavo : Flavonoïdes, Polyph : Polyphénols, Polyterp : Polyterpènes, Cat : Catéchiques, Gal : Galliques, Quin : Quinones, C. retusa : Crotalaria retusa

\section{3. Taux d'inhibition de la croissance mycélienne}

\section{3. 1. Test sur Phytophthora sp.}

L'effet de l'extrait aqueux de Crotalaria retusa sur la croissance de Phytophthora sp. comparativement au témoin, est traduit par une diminution de la croissance mycélienne en fonction de la concentration de l'extrait. Dans la boîte témoin (milieu non amendé), le fragment mycélien a atteint la périphérie de la boîte de Pétri le $7^{\text {zème }}$ jour.

Les taux d'inhibition observés dans les boîtes essais (milieux amendés), après ces 7 jours d'incubation ont été de 92,04 \pm 7,13 \% pour la concentration C1 $(0,19 \mathrm{mg} / \mathrm{ml})$, de $96,53 \pm 2,66 \%$ pour la concentration C2 $(0,39 \mathrm{mg} / \mathrm{ml})$ et de $98,41 \pm 1,33 \%$ pour la concentration C3 $(0,78 \mathrm{mg} / \mathrm{ml})$. Ces taux ont augmenté avec l'augmentation de la concentration jusqu'à la concentration C4.

Concernant les milieux C4 (1,56 mg/ml), C5 (3,12 mg/ml), C6 (6,25 $\mathrm{mg} / \mathrm{ml})$ et C7 (12,50), il n’y a pas eu de croissance ; l'inhibition a été donc totale (100\%). Toutes ces observations sont illustrées par les figures 2 et 3.

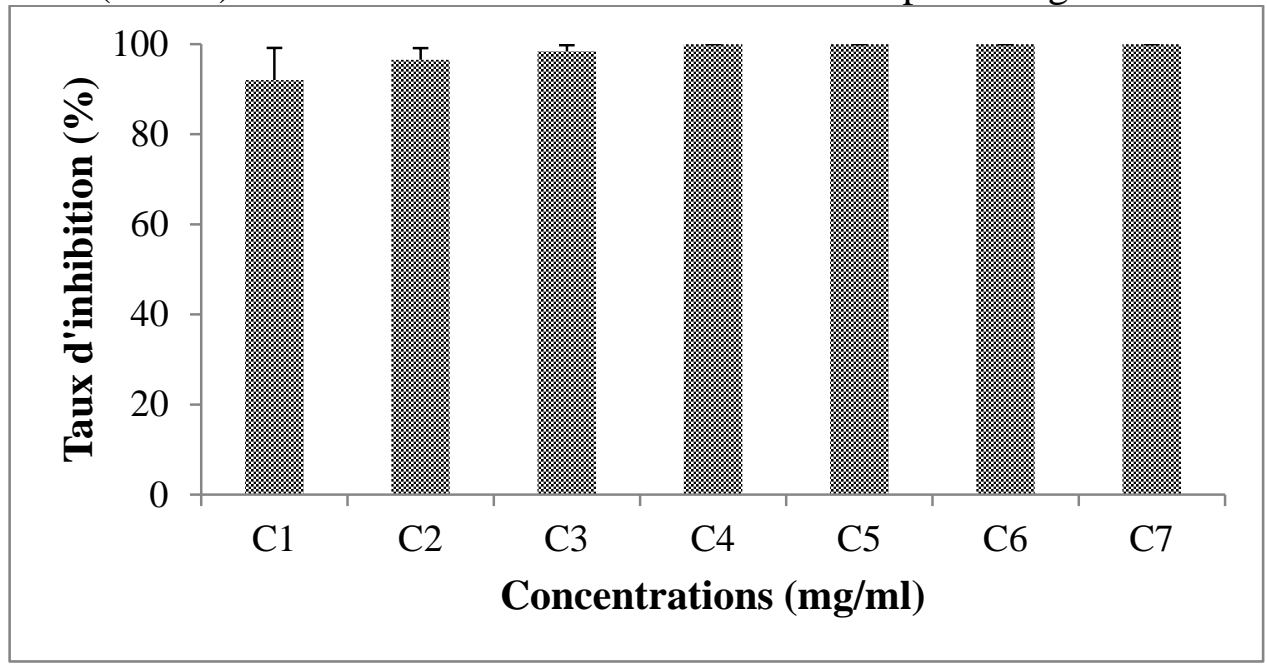

Figure 2 : Taux d'inhibition de la croissance mycélienne de Phytophthora sp. en fonction de la concentration de l'extrait aqueux des feuilles de Crotalaria retusa après 7 jours d'incubation

$\mathrm{C} 1=0,19 \mathrm{mg} / \mathrm{ml}, \mathrm{C} 2=0,39 \mathrm{mg} / \mathrm{ml}, \mathrm{C} 3=0,78 \mathrm{mg} / \mathrm{ml}, \mathrm{C} 4=1,56 \mathrm{mg} / \mathrm{ml}$, C5 = 3,12 mg/ml, C6 = 6,25 mg/ml, C7 = 12,50 mg/ml 


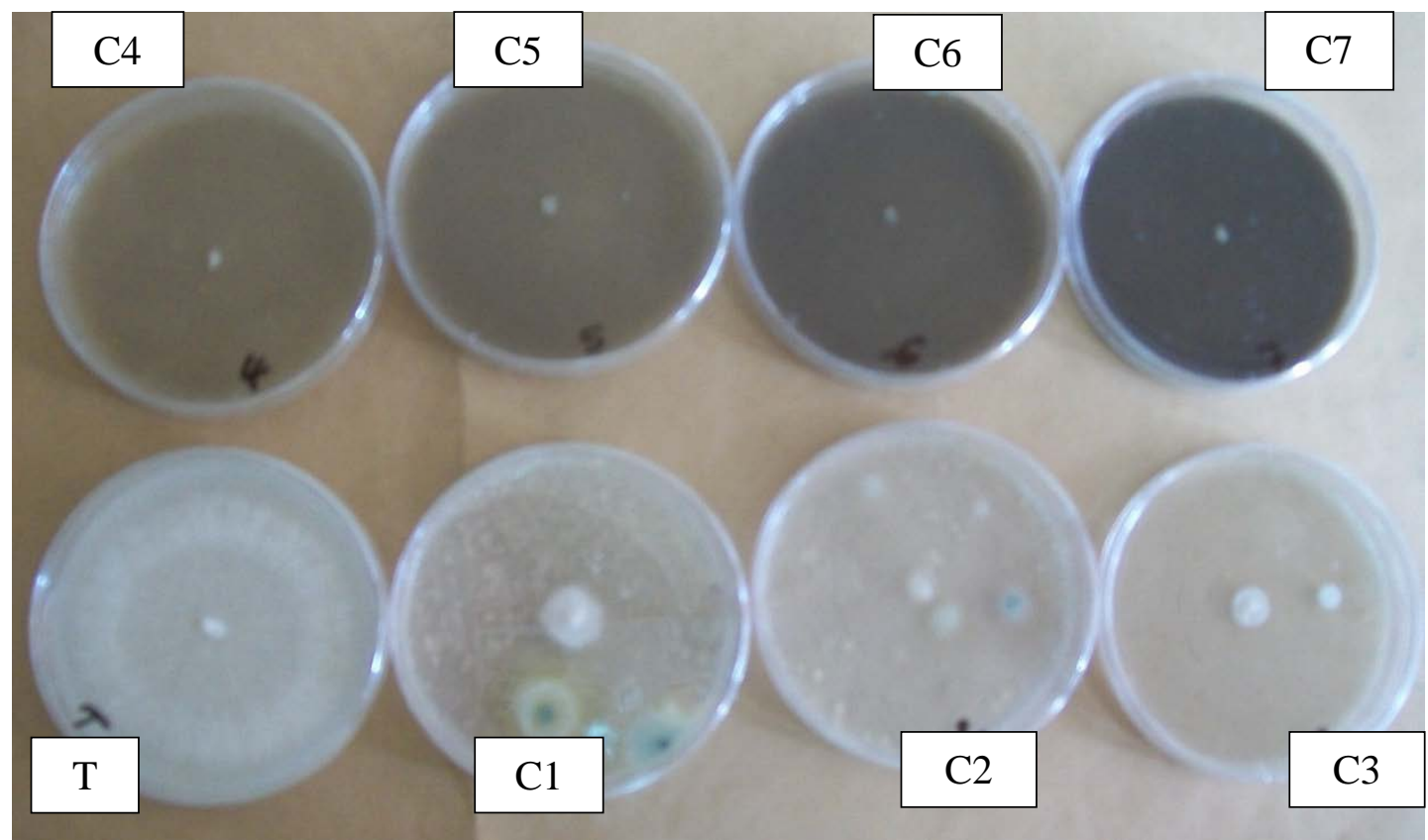

Figure 3 : Croissance in vitro du mycélium de Phytophthora sp. en présence de l'extrait aqueux des feuilles de Crotalaria retusa

$\mathrm{T}=0 \mathrm{mg} / \mathrm{ml}, \mathrm{C} 1=0,19 \mathrm{mg} / \mathrm{ml}, \mathrm{C} 2=0,39 \mathrm{mg} / \mathrm{ml}, \mathrm{C} 3=0,78 \mathrm{mg} / \mathrm{ml}, \mathrm{C} 4=1,56 \mathrm{mg} / \mathrm{ml}$

, C5 = 3,12 mg/ml, C6 = 6,25 mg/ml, C7 = 12,50 mg/ml

\section{3. 2. Test sur Fusarium solani}

L'effet de l'extrait aqueux de Crotalaria retusa sur la croissance de Fusarium solani comparativement au témoin, est traduit par une diminution de la croissance mycélienne en fonction de la concentration de l'extrait. Dans la boîte témoin (milieu non amendé), le fragment mycélien a atteint la périphérie de la boîte de Pétri le $7^{\text {ème }}$ jour.

Les taux d'inhibition observés dans les boîtes essais (milieux amendés), après ces 7 jours d'incubation ont été de 93,54 $\pm 5,49 \%$ pour la concentration C1 $(0,19 \mathrm{mg} / \mathrm{ml})$, de $97,53 \pm 1,90 \%$ pour la concentration C2 $(0,39 \mathrm{mg} / \mathrm{ml})$ et de $98,83 \pm 0,89 \%$ pour la concentration C3 $(0,78 \mathrm{mg} / \mathrm{ml})$. Ces taux ont augmenté avec l'augmentation de la concentration jusqu'à la concentration C4.

Concernant les milieux C4 (1,56 mg/ml), C5 (3,12 mg/ml), C6 (6,25 $\mathrm{mg} / \mathrm{ml})$ et C7 (12,50), il n’y a pas eu de croissance ; l'inhibition a été donc totale (100\%). Toutes ces observations sont illustrées par les figures 4 et 5 . 


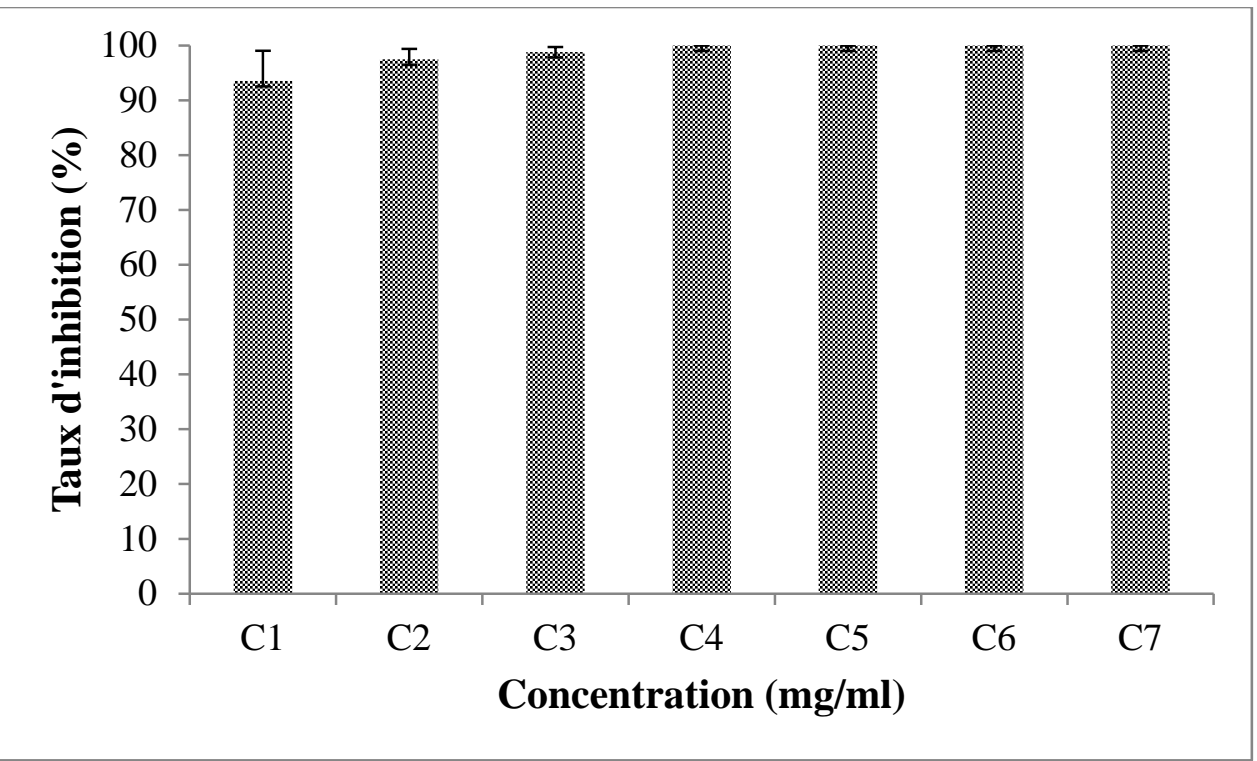

Figure 4 : Taux d'inhibition de la croissance mycélienne de Fusarium solani en fonction de la concentration de l'extrait aqueux des feuilles de Crotalaria retusa après 7 jours d'incubation

$$
\begin{gathered}
\mathrm{C} 1=0,19 \mathrm{mg} / \mathrm{ml}, \mathrm{C} 2=0,39 \mathrm{mg} / \mathrm{ml}, \mathrm{C} 3=0,78 \mathrm{mg} / \mathrm{ml}, \mathrm{C} 4=1,56 \mathrm{mg} / \mathrm{ml}, \\
\text { C5 }=3,12 \mathrm{mg} / \mathrm{ml}, \mathrm{C} 6=6,25 \mathrm{mg} / \mathrm{ml}, \mathrm{C} 7=12,50 \mathrm{mg} / \mathrm{ml}
\end{gathered}
$$

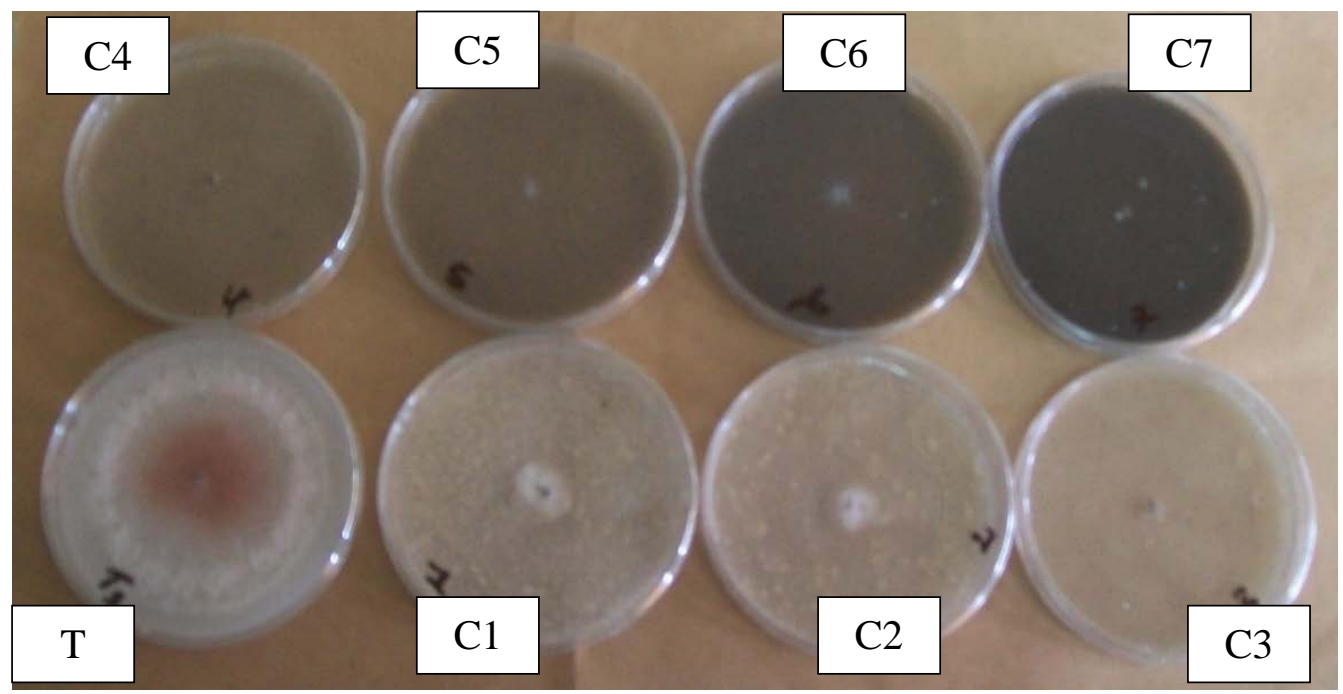

Figure 5 : Croissance in vitro du mycélium de Fusarium solani en présence d'extrait aqueux des feuilles de Crotalaria retusa

$\mathrm{T}=0 \mathrm{mg} / \mathrm{ml}, \mathrm{C} 1=0,19 \mathrm{mg} / \mathrm{ml}, \mathrm{C} 2=0,39 \mathrm{mg} / \mathrm{ml}, \mathrm{C} 3=0,78 \mathrm{mg} / \mathrm{ml}, \mathrm{C} 4=1,56 \mathrm{mg} / \mathrm{ml}$,

C5 $=3,12 \mathrm{mg} / \mathrm{ml}, \mathrm{C} 6=6,25 \mathrm{mg} / \mathrm{ml}, \mathrm{C} 7=12,50 \mathrm{mg} / \mathrm{ml}$

\section{4. Concentrations minimales inhibitrices et fongicides}

Les deux souches de champignons phytopathogènes (Phytophthora sp. et Fusarium solani) ont eu la même sensibilité vis-à-vis de l'extrait au niveau 
de la concentration minimale inhibitrice (CMI). Concernant la concentration minimale fongicide (CMF), Phytophthora sp a été plus sensible que Fusarium solani (Tableau IV).

Tableau IV : Concentrations minimales inhibitrices (CMI) et fongicides (CMF) de l'extrait aqueux des feuilles de Crotalaria retusa sur Phytophthora sp. et Fusarium solani

\begin{tabular}{lll}
\hline Mycopathogènes & CMI $(\mathbf{m g} / \mathbf{m l})$ & CMF $(\mathbf{m g} / \mathbf{m l})$ \\
\hline Phytophthora sp. & 1,56 & 6,25 \\
Fusarium solani & 1,56 & 12,50 \\
\hline
\end{tabular}

\section{DISCUSSION}

Crotalaria retusa a été la légumineuse la plus citée. Cela serait dû au fait qu'elle est utilisée dans le traitement de plusieurs affections et surtout d'origine microbienne. Ce critère a permis son choix pour le test antifongique. Dans nos investigations, plusieurs maladies ont été citées. Les plus courantes ont été les infections et le paludisme. La présence du paludisme pourrait s'expliquer par le fait que cette maladie est une parasitose qui sévit dans les régions tropicales, en particulier en Afrique subsaharienne. Le paludisme est considéré comme une cause de la pauvreté (OMS, 2004). Quant aux infections, elles seraient attribuées au manque d'hygiène et à la pauvreté.

Le tri phytochimique effectué sur l'extrait aqueux de Crotalaria retusa, a indiqué la présence de tanins, de polyphénols, de quinones, de stérols, de polyterpènes, de flavonoïdes, de saponosides et d'alcaloïdes. Ces molécules pourraient justifier l'utilisation de cette plante dans la pharmacopée traditionnelle. En effet, selon plusieurs auteurs, ces métabolites secondaires possèdent des activités antifongiques, antibactériennes et antiparasitaires (Frankel, 1993 ; Haluk et al., 2001 ; Olajidé et al., 2003 ; Zirihi et al., 2005 ; 2006 ; 2007 ; Okigbo et Ajalie, 2005 ; N’Guessan et al., 2009 ; Mezouar et al., 2014).

Le pouvoir inhibiteur de l'extrait aqueux sur la croissance du mycélium a été mis en évidence par la réduction de la croissance mycélienne avec l'augmentation de la concentration de l'extrait. L'effet inhibiteur de l'extrait $(\mathrm{CMI}=1,56 \mathrm{mg} / \mathrm{ml})$ a été le même pour les deux champignons phytopathogènes (Phytophthora sp. et Fusarium solani). Mais concernant le pouvoir fongicide, les deux champignons ont eu des réactions différentes $(\mathrm{CMF}=6,25 \mathrm{mg} / \mathrm{ml}$ pour Phytophthora $\mathrm{sp}$. et $\mathrm{CMF}=12,50 \mathrm{mg} / \mathrm{ml}$ pour Fusarium solani). Phytophthora sp. a été donc plus sensible. Des études similaires ont été conduites par Orsot et al. (2015). Ces auteurs ont montré l'effet antifongique des extraits des feuilles de Mallotus oppostifolius sur deux souches phytopathogènes de Sclerotium rolfsii. 


\section{CONCLUSION}

La légumineuse Crotalaria retusa a été sélectionnée parmi 11 espèces de légumineuses identifiées à l'issue d'une enquête ethnobotanique réalisée dans la Sous-préfecture de Lauzoua. Cette enquête avait pour objet d'identifier les légumineuses locales d'intérêt thérapeutique afin de les valoriser dans les biotechnologies agricoles. Selon cette étude, Crotalaria retusa passe pour être la légumineuse la plus utilisée dans le traitement des maladies microbiennes. Ce critère a permis de tester l'activité antifongique de cette plante sur deux champignons phytopathogènes (Phytophthora sp. et Fusarium solani). L'extrait aqueux de cette plante a eu une activité antifongique sur ces deux pathogènes. Cet extrait a été fongicide à 6,25 $\mathrm{mg} / \mathrm{ml}$ sur Phytophthora $s p$. et $12,50 \mathrm{mg} / \mathrm{ml}$ sur Fusarium solani. La présence des composés chimiques mise en évidence pourrait justifier l'activité antifongique de cette légumineuse et son utilisation en milieu traditionnel. L'extrait de Crotalaria retusa pourrait donc servir de biofongicide contre des champignons phytopathogènes.

\section{REMERCIEMENTS}

Nous tenons à remercier Monsieur N'Guessan Amani Antoine, Botaniste au Département FOREN (Foresterie et Environnement) de l'Institut National Félix HOUPHOUËT-BOIGNY pour sa contribution à l'identification des légumineuses ; la famille DEMBELE pour notre séjour à Lauzoua et le Groupe de Recherche Chimie de l'Eau et des Substances Naturelles (GCESNA) de l'Institut National Félix HOUPHOUËT-BOIGNY pour le tri phytochimique.

\section{Références}

1. Ahon M.G., Akapo-Akue J.M., Kra M.A., Ackab J.B., Zirihi N.G., Djaman J.A., 2011. Antifungal activity of the aqueous and hydroalcoholic extracts of Terminalia superba Engl. On the in vitro growth of clinical isolates of pathogenic fungi. Agric. Biol.J.N. Am., 2 (2) : 250-257.

2. Assigbetse K., Henni J., Boisson C., 1990. Pathogenicity variations of three pathogens of tomato and cotton : Verticillium Dahliaklebahn, Fusarium Oxysporum f sp. Lycopercisi (Sacc.) Snyd. and Hans. and F. Oxysporum f. sp. Vasinfectum (Atk.) Snyd. and Hans. Report of the $8^{\mathrm{e}}$ Congress of the Mediterranean Phytopathological Union, Agadir-Morocco, 381-383.

3. Bruneton J., 2009. Pharmacognosie, Phytochimie, Plantes médicinales. Paris, $4^{\mathrm{e}}$ Edition Lavoisier. 
4. Drame A., 2004. Pathogénie comparée de quelques souches de Fusarium oxysporum f. sp. vasinfectum (Atk.), agent de la fusariose du gombo (Abelmoschus esculentus L.) au Sénégal. Agron Afr., 16 (2) : 33-38.

5. Flood J., 2006. The threat from global spread of cocoa pests and diseases : hypothetical scenario or clear and present danger. In: proceedings of the $15^{\text {th }}$ International Cocoa Research Conference, 910 October 2006. San Jose (Costa Rica), 857-872.

6. Frankel E.N., Kanner J., German J.B., Parks E., Kinsella J.E., 1993. Inhibition of oxidation of human low-density lipoprotein by phenolic substances in red wine. In The Lancet, 341 (8843) : 454-457.

7. Haluk J.P., Bousta-Roussel C., Thévenon M.F., 2001. Importance des quinones dans les propriétés antifongiques du teck (Tectona grandis). In : Les cahiers scientifiques du bois, Nancy : Association pour la recherche sur le bois en Lorraine, 2 : 77-83.

8. Hibar K., Medja D., Mahjoub M.E., 2007. Effect of some chemical and biological fungicides on mycelial growth and disease severity of Fusarium oxysporum f sp racidislycopersici. Tropicultura, 25 (3) : 146-1452.

9. Kouamé K. D., 2006. Structure et dynamique des populations de Phytophthora spp., agents de la pourriture brune des cabosses du cacaoyer (Theobroma cacao L.) en Côte d'Ivoire. Mémoire de DEA. UFR Biosciences. Université de Cocody, Abidjan, Côte d’Ivoire, 74 p.

10. Lass R. A., 1985. Disease. In Cocoa.. Wood G.A.R., \& Lass R. A. Eds. $4^{\text {th }}$ Edition, Longman, Longman, 265-365.

11. Lauzon M., Dion Y., Rioux S., 2007. Fusariose de l'épi chez le blé et l'orge. CEROM, Bulletin Technique, Phytopathologie, $\mathrm{N}^{\mathrm{O}}$ 2. 01, Québec (Canada).

12. Leroux P. et Credet A., 1978. Document sur l'étude de l'activité des fongicides. INRA. Versailles France, 12 p.

13. Mangambu M., Mushagalusa K., Kadima N., 2014. Contribution à l'étude phytochimique de quelques plantes médicinales antidiabétiques de la ville Bukavu et ses environs (Sud-Kivu, R. D. Congo).Journal of Applied Biosciences, 75 : 6211-6220.

14. DOI: http://dx.doi.org/10.4314/jab.v75i1.7.

15. Mezouar D., Lahfa F.B., Abdelouahid D.E., Adida H., Rahmoun N.M., Boucherit Z., Otmani, 2014. Activité antimicrobienne d'extraits d'écorce de racine de Berberis vulgaris. Springer, 12 (6) : $380 \mathrm{p}$.

16. Moore N.Y., Bentley S., Pegg K.G., Jones R.D., 1995. Maladies des Musa. Fiche technique $\mathrm{N}^{\circ} 5$. La fusariose du bananier, Réseau 
international pour l'amélioration de la banane et de la banane plantain. Montpellier : Parc Scientifique Agropolis.

17. N’Guessan K, Kadja B, Zirihi G.N, Traoré D, Aké-Assi L., 2009. Screening phytochimique de quelques plantes médicinales ivoiriennes. Sciences \&Nature, $6: 1-15$.

18. Okigbo R. et Ajalie A., 2005. Inhibition of some human pathogens with tropical plants extracts Chromolinaena odorata and citrus aurantifolia and some antibiotics. Inter. J. Mol. Med. Adv. Sci., 1 (1) : 34-40.

19. Olajide O.A., Okpako D.T., Makinde J.M., 2003. Ethnopharmacol J., $88(2-3)$ : 221-224.

20. OMS, 2004. Le paludisme, une des principales causes de décès et de pauvreté des enfants en Afrique. http://rbm.who.int/docs/rps_publications/unicef_malaria_fr. pdf.

21. Orsot B.A.M.B., Soro S., Ouattara D., N'guessan E.K., Zirihi G.N., 2015. Etude ethnobotanique et évaluation in vitro de l'activité antifongique des extraits de feuilles de Mallotus oppositifolius sur deux souches phytopathogènes de Sclerotium rolfsii. European Scientific Journal. Edition, 11 (36) : issn : 1857 - 7881 (print)e - issn 1857- 7431.

22. Zehhar G., Touhami A. O., Badoc A., Douira A., 2006. Effet des Fusarium des eaux de rizière sur la germination et la croissance des plantules de riz. Bull. Soc. Pharm., 145 : 7-18.

23. Zirihi G.N., Kra A.K.M., Guédé-Guina F., 2003. Evaluation de l'activité antifongique de Microglossa pyrifolia (Lam) O. Ktze (Asteraceae) «PYMI» sur la croissance in vitro de Candida albicans. Revue Médicale et Pharm. Afric, 17 : 1-19.

24. Zirihi G.N., Grellier P., Guédé-Guina F., Bodo B., Lengo M., 2005. Isolation, Characterisation and antiplasmodial activity of steroidal alkaloids from Funtumia elastic (Preuss) Stapf. Biorganic and Medicinal Chemistry Letters, 15 : 2637-2640.

25. Zirihi G.N., 2006. Etudes botanique, pharmacologique et phytochimique de quelques plantes Médicinales antipaludiques et/ou immunogènes utilisées chez les Bété du Département d’Issia, dans l'ouest de la Côte d'Ivoire. Thèse de Doctorat d'Etat, Université de Cocody Abidjan, UFR Biosciences, 126 p.

26. Zirihi G.N., Datté J.Y., Kra-Adou K.M., Grellier P., 2007. Phytochemical and pharmacological studies of the alcoholic extract (MFA) of Fagara macrophylla (Oliv.) Engl. (Rutaceae) : the chemical structure of the active compound inducing antipaludic activity. Journal of Chinese Clinical Medicine, 2 : 205-210. 